\title{
FACTORIZATION OF MATRICES INTO PARTIAL ISOMETRIES
}

\author{
KUNG-HWANG KUO AND PEI YUAN WU
}

(Communicated by John B. Conway)

\begin{abstract}
In this paper, we characterize complex square matrices which are expressible as products of partial isometries and orthogonal projections. More precisely, we show that a matrix $T$ is the product of $k$ partial isometries $(k \geq 1)$ if and only if $T$ is a contraction $(\|T\| \leq 1)$ and rank $\left(1-T^{*} T\right) \leq$ $k \cdot$ nullity $T$. It follows, as a corollary, that any $n \times n$ singular contraction is the product of $n$ partial isometries and $n$ is the smallest such number. On the other hand, $T$ is the product of finitely many orthogonal projections if and only if $T$ is unitarily equivalent to $1 \oplus S$, where $S$ is a singular strict contraction $(\|S\|<1)$. As contrasted to the previous case, the number of factors can be arbitrarily large.
\end{abstract}

\section{INTRODUCTION}

An $n \times n$ complex matrix $T$ is a partial isometry if $\|T x\|=\|x\|$ for any vector $x$ in $\operatorname{ker}^{\perp} T$, the orthogonal complement of the kernel of $T$ in $\mathbf{C}^{n}$, where $\|x\|$ denotes the 2-norm $\|x\|=\left(\sum_{i=1}^{n}\left|x_{i}\right|^{2}\right)^{1 / 2}$ of $x=\left[x_{1} \cdots x_{n}\right]^{t}$ in $\mathbf{C}^{n}$. Examples of partial isometries are (orthogonal) projections $\left(T^{2}=T=T^{*}\right)$ and unitary matrices $\left(T^{*}=T^{-1}\right)$. In this paper, we will characterize matrices which are expressible as products of partial isometries and projections.

As we will show below, the situations for these two types of products are quite different. For the former, we obtain that $T$ is the product of $k$ partial isometries $(k \geq 1)$ if and only if $T$ is a contraction $(\|T\| \leq 1)$ and rank $\left(1-T^{*} T\right) \leq k \cdot$ nullity $T$ (Theorem 2.2). This latter condition links our problem to that of factorization into idempotent matrices (cf. [1]). In particular, it follows that any $n \times n$ singular contraction is the product of $n$ partial isometries and $n$ is the smallest such number (Corollary 2.4). (Recall that a matrix is singular if it does not have an inverse.)

Products of partial isometries have also been considered before by Erdelyi [3]. However his concern is different from ours. He was interested in conditions under which a product of partial isometries is itself a partial isometry.

Received by the editors August 13, 1987 and, in revised form, January 19, 1988.

1980 Mathematics Subject Classification (1985 Revision). Primary 15A23.

Key words and phrases. Partial isometry, orthogonal projection, contraction, idempotent matrix.

Research of the second author was partially supported by the National Science Council of the Republic of China. 
As for products of projections, very few seem to be known in the literature. One exception is the characterization of products of two projections due to Crimmins (cf. [5, Theorem 8]) which is true even for bounded linear operators on infinite-dimensional Hilbert spaces: $T$ is such a product if and only if $T T^{*} T=T^{2}$. In this paper, we characterize products of finitely many projections. More precisely, we will show that a matrix $T$ is such a product if and only if $T$ is unitarily equivalent to $1 \oplus S$, where $S$ is a singular strict contraction $(\|S\|<1)$ (Theorem 3.1). Note the similarity of this result to that for partial isometries: $T$ is the product of finitely many partial isometries if and only if $T$ is unitarily equivalent to $U \oplus S$, where $U$ is unitary and $S$ is a singular contraction (Corollary 2.3). However, there is one big difference between these two types of products: unlike the partial isometry products, the number of projections in a product can be arbitrarily large.

\section{PARTIAL ISOMETRY}

We start with the following simple observation.

Lemma 2.1. If $T$ is a partial isometry and $U$ is unitary, then $U T$ and $T U$ are also partial isometries.

Proof. This follows from the fact that a matrix $S$ is a partial isometry if and only if $S S^{*} S=S$ (cf. [4, Corollary 3 to Problem 127]). It is also a consequence of [3, Theorem 1].

The preceding lemma reduces, via the singular-value decomposition, the partial isometry factorization of arbitrary matrices to that of positive semidefinite ones. In the following, nullity $T$ denotes the dimension of $\operatorname{ker} T$. A matrix $T$ is idempotent if $T^{2}=T$.

Theorem 2.2. Let $T$ be an $n \times n$ matrix and $k \geq 1$. Then the following statements are equivalent:

(1) $T$ is the product of $k$ partial isometries;

(2) $\|T\| \leq 1$ and $\operatorname{rank}\left(1-T^{*} T\right) \leq k \cdot$ nullity $T$;

(3) $\|T\| \leq 1$ and $\left(T^{*} T\right)^{1 / 2}$ is the product of $k$ idempotent matrices.

Proof. (1) $\Rightarrow(2)$. Let $T=A_{1} A_{2} \cdots A_{k}$ be the product of $k$ partial isometries. Since the norm of any nonzero partial isometry is one, we have $\|T\| \leq 1$. Next let $K=\left\{x \in \mathbf{C}^{n}: T^{*} T x=x\right\}$. We claim that

$$
\begin{aligned}
K= & \operatorname{ker}^{\perp} A_{k} \cap A_{k}^{-1}\left(\operatorname{ker}^{\perp} A_{k-1}\right) \\
& \cap \cdots \cap A_{k}^{-1}\left(A_{k-1}^{-1}\left(\cdots\left(A_{2}^{-1}\left(\operatorname{ker}^{\perp} A_{1}\right)\right) \ldots\right)\right) .
\end{aligned}
$$

Indeed, if $x \in K$, then $T^{*} T x=x$ whence $\|T x\|^{2}=\left(T^{*} T x, x\right)=(x, x)=$ $\|x\|^{2}$. For each $j=1,2, \ldots, k$, let $A_{j+1} \cdots A_{k} x=y_{1}+y_{2}$, where $y_{1} \in \operatorname{ker} A_{j}$ 
and $y_{2} \in \operatorname{ker}^{\perp} A_{j}$. Since

$$
\begin{aligned}
\left\|y_{1}\right\|^{2}+\left\|y_{2}\right\|^{2} & =\left\|A_{j+1} \cdots A_{k} x\right\|^{2} \leq\|x\|^{2} \\
& =\|T x\|^{2}=\left\|A_{1} A_{2} \cdots A_{k} x\right\|^{2} \\
& =\left\|A_{1} \cdots A_{j} y_{2}\right\|^{2} \leq\left\|y_{2}\right\|^{2},
\end{aligned}
$$

we infer that $y_{1}=0$ or, equivalently, $x \in A_{k}^{-1}\left(A_{k-1}^{-1}\left(\cdots\left(A_{j+1}^{-1}\left(\operatorname{ker}^{\perp} A_{j}\right)\right) \cdots\right)\right)$. This shows that $x$ belongs to the right hand side of $(*)$. Conversely, if $x$ belongs to this subspace, then $A_{j+1} \cdots A_{k} x \in \operatorname{ker}^{\perp} A_{j}$ for each $j=1,2, \ldots, k$. Hence

$$
\left\|A_{j} A_{j+1} \cdots A_{k} x\right\|=\left\|A_{j+1} \cdots A_{k} x\right\| \text { for each } j .
$$

Therefore, $\|T x\|=\|x\|$. This implies that $\left(T^{*} T x, x\right)=(x, x)$ or $((1-$ $\left.\left.T^{*} T\right) x, x\right)=0$. Since $0 \leq T^{*} T \leq 1$, we may consider the positive square root of $1-T^{*} T$ and obtain

$$
\left\|\left(1-T^{*} T\right)^{1 / 2} x\right\|^{2}=\left(\left(1-T^{*} T\right) x, x\right)=0 .
$$

Thus $\left(1-T^{*} T\right)^{1 / 2} x=0$ which implies that $\left(1-T^{*} T\right) x=0$ or $T^{*} T x=x$. This proves $(*)$.

To conclude the proof of $(1) \Rightarrow(2)$, let $m=$ nullity $T$. Then $\operatorname{rank} A_{j} \geq$ $n-m$ for each $j$. It is easily seen that

$$
\begin{aligned}
A_{k} K= & \operatorname{ran} A_{k} \cap \operatorname{ker}^{\perp} A_{k-1} \cap A_{k-1}^{-1}\left(\operatorname{ker}^{\perp} A_{k-2}\right) \\
& \cap \cdots \cap A_{k-1}^{-1}\left(\cdots\left(A_{2}^{-1}\left(\operatorname{ker}^{\perp} A_{1}\right)\right) \cdots\right) .
\end{aligned}
$$

Hence

$$
\begin{aligned}
\operatorname{dim} K & \geq \operatorname{dim} A_{k} K \\
& \geq \operatorname{rank} A_{k}+\operatorname{dim}\left(\operatorname{ker}^{\perp} A_{k-1} \cap A_{k-1}^{-1}\left(\operatorname{ker}^{\perp} A_{k-2}\right) \cap \cdots\right. \\
& \geq \cdots \\
& \left.\cap A_{k-1}^{-1}\left(\cdots\left(A_{2}^{-1}\left(\operatorname{ker}^{\perp} A_{1}\right)\right) \cdots\right)\right)-n \\
& \geq \sum_{j=1}^{k} \operatorname{rank} A_{j}-(k-1) n \\
& \geq k(n-m)-(k-1) n=n-k m .
\end{aligned}
$$

On the other hand, we also have

$$
\operatorname{dim} K=\operatorname{nullity}\left(1-T^{*} T\right)=n-\operatorname{rank}\left(1-T^{*} T\right) .
$$

Hence $\operatorname{rank}\left(1-T^{*} T\right) \leq k m$ as asserted.

(2) $\Rightarrow(1)$. Let $T=U P V$ be the singular-value decomposition of $T$, where $U$ and $V$ are unitary and $P=\operatorname{diag}\left(a_{1}, \ldots, a_{n}\right)$ is the diagonal matrix with the singular values $1 \geq a_{1} \geq \cdots \geq a_{n} \geq 0$ of $T$ on its diagonal. By Lemma 2.1, it suffices to factor $P$ into $k$ partial isometries. Let $l=\operatorname{rank}\left(1-T^{*} T\right)$ and 
$m=$ nullity $T$. In terms of the singular values, this says that $a_{1}=\cdots=a_{n-l}=$ $1,0<a_{n-l+1}, \ldots, a_{n-m}<1$ and $a_{n-m+1}=\cdots=a_{n}=0$. We only need factor the $l \times l$ matrix $P^{\prime}=\operatorname{diag}\left(a_{n-l+1}, \ldots, a_{n-m}, 0, \ldots, 0\right)$. Let $l-m=2 m s+t$, where $0 \leq t<2 m$ and let $r=s$ or $s+1$ depending on whether $t=0$ or $t>0$. Then we have $P^{\prime}=P_{1} P_{2} \ldots P_{r}$, where $P_{j}, j=1,2, \ldots, r$, is the diagonal matrix obtained from $P^{\prime}$ by retaining $a_{n-l+2(j-1) m+1}, \ldots, a_{n-l+2 j m}$ and replacing the remaining nonzero diagonal entries by 1 's. Note that, other than $P_{r}$, each $P_{j}$ can be written as

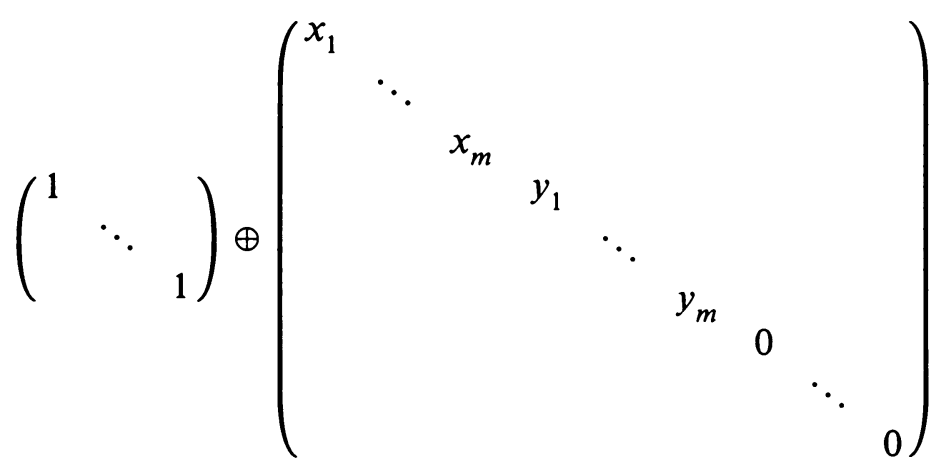

whose second summand is the product of three partial isometries

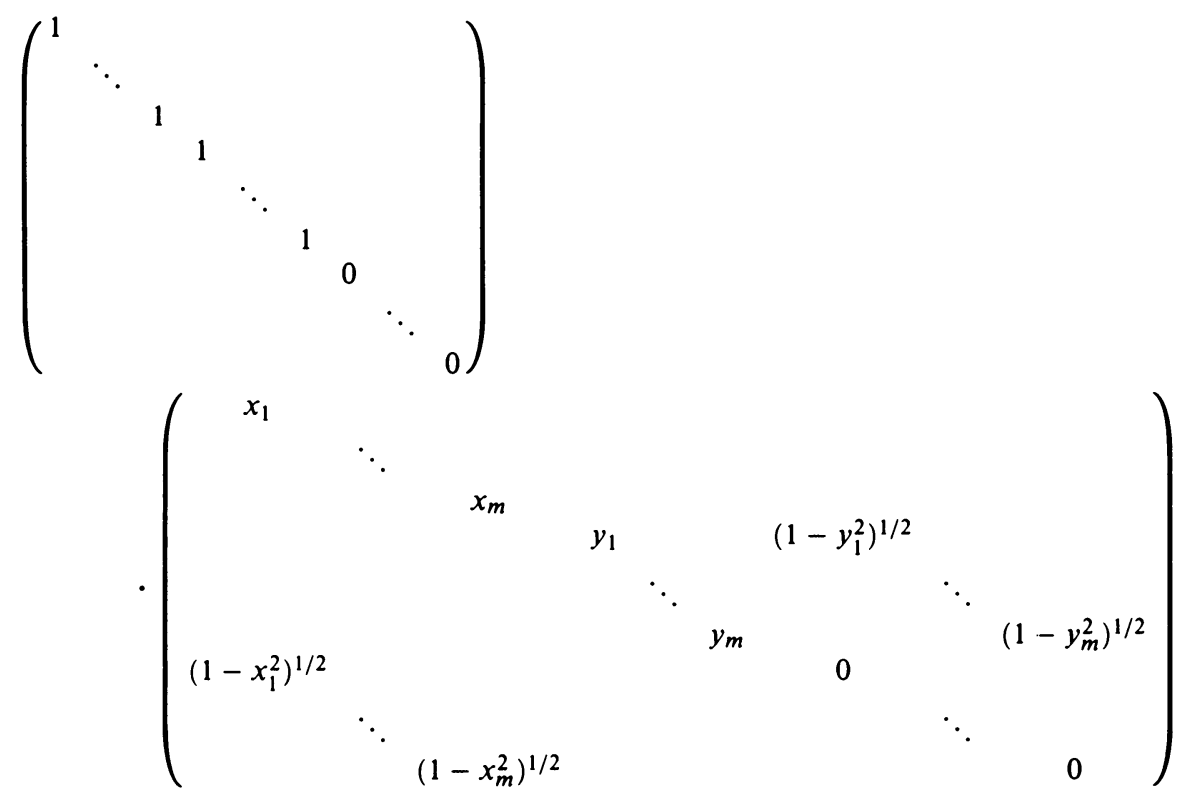




$$
\cdot\left(\begin{array}{ccccccccc}
1 & & & & & & & & \\
& \ddots & & & & & & & \\
& & 1 & & & & & & \\
& & & 1 & & & & & \\
& & & & \ddots & & & & \\
& & & & & 1 & & & \\
& & & & & & 0 & & \\
& & & & & & & \ddots & \\
& & & & & & & & 0
\end{array}\right) .
$$

Hence the same is true for each $P_{j}, j=1, \ldots, r-1$, say, $P_{j}=J Q_{j} J$, where $Q_{j}$ is a partial isometry and $J$ denotes the matrix

$$
\operatorname{diag}(\underbrace{1, \ldots, 1}_{l-m}, \underbrace{0, \ldots, 0}_{m}) .
$$

Similar arguments applied to $P_{r}$ yields that $P_{r}=J Q_{r} J$ or $J Q_{r}$ depending on whether $t>m$ or $\leq m$. In the former case, we have

$$
P^{\prime}=\left(J Q_{1} J\right) \cdots\left(J Q_{r-1} J\right)\left(J Q_{r} J\right)=J Q_{1} J Q_{2} \cdots J Q_{r} J
$$

with $2 r+1$ factors. Since $l \leq k m$, we have $(l-m) / 2 m \leq 1 / 2(k-1)$. If $k$ is odd, say, $k=2 q+1$, then $s+t / 2 m \leq q$ whence $r=s+1 \leq q$ and we have $2 r+1 \leq 2 q+1=k$ as required. If $k$ is even, say $k=2 q$, then $s+t / 2 m \leq q-1 / 2$ and, since $t>m$, we have $s+2 \leq q$ which implies that $2 r+1=2 s+3 \leq 2 q-1 \leq k-1$ as required. Analogously, for $t \leq m$ we can prove that $P^{\prime}$ is the product of $2 r$ partial isometries and $2 r \leq k$.

The equivalence of (2) and (3) follows from the main theorem in [1]. This completes the proof.

Here are some immediate corollaries.

Corollary 2.3. A complex square matrix is the product of finitely many partial isometries if and only if it is either unitary or a singular contraction.

Corollary 2.4. Any $n \times n$ singular contraction is the product of $n$ partial isometries and there are such matrices which are not the product of $n-1$ partial isometries.

Proof. The assertions follow from Theorem 2.2 and from considering matrices of the form $\operatorname{diag}\left(a_{1}, \ldots, a_{n-1}, 0\right)$, where $\left|a_{i}\right|<1$ for $i=1, \ldots, n-1$.

We remark in passing that on an infinite-dimensional Hilbert space, every contraction is the product of two partial isometries. More precisely, a contraction $T$ can be factored as $S_{1}^{*} S_{2}$, where $S_{1}$ and $S_{2}$ are unilateral shifts with infinite multiplicity (cf. [2]).

\section{Projection}

The main result of this section is the following characterization of products of projections. 
Theorem 3.1. An $n \times n$ matrix $T$ is the product of finitely many projections if and only if $T$ is unitarily equivalent to $1 \oplus S$, where $S$ is singular with $\|S\|<1$. Moreover, for each $n \geq 2$, the number of projections in such a factorization can be arbitrarily large.

Proof of necessity. Assume that $T=P_{1} P_{2} \ldots P_{m}$, where $P_{j}$ 's are projections. Then $T$ is a contraction and the subspace $K=\{x: T x=x\}$ reduces $T$ (cf. [6, p. 8]). Let $T=1 \oplus S$ with respect to the decomposition $K \oplus K^{\perp}$. Note that if $x$ is any vector satisfying $\|T x\|=\|x\|$, then $x$ must be in $K$. Indeed, from

$$
\|x\|=\|T x\|=\left\|P_{1} P_{2} \cdots P_{m} x\right\| \leq\left\|P_{2} \cdots P_{m} x\right\| \leq \cdots \leq\left\|P_{m} x\right\| \leq\|x\|
$$

we infer that

$$
\left\|P_{1} P_{2} \cdots P_{m} x\right\|=\left\|P_{2} \cdots P_{m} x\right\|=\cdots=\left\|P_{m} x\right\|=\|x\| .
$$

The last equality $\left\|P_{m} x\right\|=\|x\|$ implies that $P_{m} x=x$. Then from $\left\|P_{m-1} P_{m} x\right\|$ $=\|x\|$ we have $\left\|P_{m-1} x\right\|=\|x\|$ which implies that $P_{m-1} x=x$. Arguing successively, we obtain that $P_{j} x=x$ for all $j$ whence $T x=P_{1} P_{2} \cdots P_{m} x=x$ as asserted. Note that if $\|S\|=1$, then there exists a unit vector $x$ in $K^{\perp}$ such that $\|S x\|=\|T x\|=1$. From above we have $x \in K$. This together with $x \in K^{\perp}$ implies that $x=0$, a contradiction. Thus we must have $\|S\|<1$. That $S$ is singular is trivial.

To prove the sufficiency, we start with the following two elementary lemmas whose proofs we omit.

Lemma 3.2. (1) For any real $\theta$ and $\alpha$,

$$
P(\theta, \alpha)=\left(\begin{array}{cc}
\cos ^{2} \theta & \sin \theta \cos \theta e^{i \alpha} \\
\sin \theta \cos \theta e^{-i \alpha} & \sin ^{2} \theta
\end{array}\right)
$$

is the projection onto the subspace of $\mathbf{C}^{2}$ generated by

$$
\left(\begin{array}{c}
\cos \theta e^{i \alpha} \\
\sin \theta
\end{array}\right)
$$

(2) Any $2 \times 2$ projection with rank 1 is of the form $P(\theta, \alpha)$ for some $\theta$ and $\alpha$.

Lemma 3.3. (1) For any $0<\theta \leq \frac{1}{2} \pi,(\cos (\theta / n))^{n}$ is strictly increasing with limit 1 as $n$ approaches infinity.

(2) $\prod_{j=1}^{n}\left|\cos \theta_{j}\right| \leq(\cos (\pi / 2 n))^{n}$ for any real $\theta_{1}, \ldots, \theta_{n}$ satisfying $\sum_{j=1}^{n} \theta_{j}$ $=\frac{1}{2} \pi$.

Our next lemma is an easy observation. It holds even for operators on infinitedimensional spaces. 
Lemma 3.4. If $T=P_{1} P_{2} \ldots P_{m}$ is the product of $m(\geq 2)$ projections, then $T=Q P_{2} \cdots P_{m-1} R$, where $Q$ and $R$ are the projections onto the subspaces $\operatorname{ran} T$ and $\operatorname{ker}^{\perp} T$, respectively.

Proof. Since $T=P_{1} P_{2} \ldots P_{m}$ implies that $\operatorname{ran} T \subseteq \operatorname{ran} P_{1}$ and $\operatorname{ker}^{\perp} T \subseteq$ ran $P_{m}$, we have $Q P_{1}=Q$ and $P_{m} R=R$. Thus $T=Q T R=Q P_{1} P_{2} \cdots P_{m} R=$ $Q P_{2} \cdots P_{m-1} R$ as asserted.

The next result is, in nature, a two-dimensional one. It is the main step toward our sufficiency proof and may have some independent interest.

Lemma 3.5. Let $x$ and $y$ be vectors in $\mathbf{C}^{n}$. Then a necessary and sufficient condition that $x=P_{1} \cdots P_{m} y$ for some projections $P_{1}, \ldots, P_{m}$ is that either $x=y$ or $\|x\|<\|y\|$. Moreover, in this case, the $P_{j}$ 's may be chosen to fix all the vectors which are orthogonal to a fixed two-dimensional subspace containing $x$ and $y$.

Proof. If $x=P_{1} \cdots P_{m} y$ and $\|x\|=\|y\|$, then, as proved in the necessity part of Theorem 3.1, $x=y$. To prove the converse, let $x$ and $y$ be such that $\|x\|<\|y\|$. By restricting to a fixed two-dimensional subspace containing $x$ and $y$, changing the scale and rotating this subspace appropriately, we may assume that $x=\left(\begin{array}{l}a \\ b\end{array}\right)$ with $0<|a|^{2}+|b|^{2}<1$ and $y=\left(\begin{array}{l}0 \\ 1\end{array}\right)$. We consider the following four cases successively:

(1) $|a|^{2}+|b|^{2}=b$. In this case, $x=P y$, where

$$
P=\left(\begin{array}{cc}
|a|^{2} / b & a \\
\bar{a} & b
\end{array}\right)
$$

(2) $|a|^{2}+|b|^{2}<b$. Let $P$ be the projection from $\mathbf{C}^{2}$ onto the subspace generated by $x$, let $s$ and $t$ be a pair of positive solutions of the equations $s^{2}+t^{2}=t$ and $(s-|a|)|a|+(t-b) b=0$, and let $c=s a /|a|$ if $a \neq 0$ and $s$ if $a=0$, and $d=t$. Then it is easily seen that $x=P\left(\begin{array}{l}c \\ d\end{array}\right)$. Since $|c|^{2}+|d|^{2}=d$, (1) yields that $\left(\begin{array}{l}c \\ d\end{array}\right)=P^{\prime} y$ for some projection $P^{\prime}$. Hence $x=P P^{\prime} y$ as required.

(3) $|a|^{2}+|b|^{2}>b \geq 0$. Let $r=\left(|a|^{2}+|b|^{2}\right)^{1 / 2}$ and $\theta=\tan ^{-1} \frac{b}{|a|}$. By Lemma 3.3(1), there exists an integer $N$ such that $r\left(\sec (1 / N)\left(\frac{1}{2} \pi-\theta\right)\right)^{N}<1$. Let $\eta=(1 / N)\left(\frac{1}{2} \pi-\theta\right)$ and $\theta_{j}=\theta+(j-1) \eta$ for $j=1,2, \ldots, N$. Let $a_{0}=a$, $b_{0}=b$, and, for $j=1,2, \ldots, N$, let

$$
a_{j}=r(\sec \eta)^{j-1} \cos \theta_{j} \frac{a_{j-1}}{\left|a_{j-1}\right|}
$$

and

$$
b_{j}=r(\sec \eta)^{j-1} \sin \theta_{j} .
$$

Note that $a_{1}=a$ and $b_{1}=b$. Let $P_{j}=P\left(\theta_{j}, \arg a_{j-1}\right)$ be the projection onto the subspace generated by $\left(\cos \theta_{j} \frac{a_{j-1}}{\left|a_{j-1}\right|} \sin \theta_{j}\right)^{t}, j=1,2, \ldots, N-1$, or, 
equivalently, by $\left(\begin{array}{l}a_{j} \\ b_{j}\end{array}\right)$. It is easily seen that $\left(\begin{array}{l}a_{j} \\ b_{j}\end{array}\right)=P_{j}\left(\begin{array}{c}a_{j+1} \\ b_{j+1}\end{array}\right)$ for $j=1,2, \ldots, N-$ 1 . Hence we have $x=P_{1} P_{2} \cdots P_{N-1}\left(\begin{array}{l}a_{N} \\ b_{N}\end{array}\right)$. Since

$$
\left(\left|a_{N}\right|^{2}+\left|b_{N}\right|^{2}\right)^{1 / 2}=r(\sec \eta)^{N-1}<\cos \eta=\sin \theta_{N}=b_{N} /\left(\left|a_{N}\right|^{2}+\left|b_{N}\right|^{2}\right)^{1 / 2},
$$

that is, $\left|a_{N}\right|^{2}+\left|b_{N}\right|^{2}<b_{N}$, it follows from (2) that there exist projections $P$ and $P^{\prime}$ such that

$$
\left(\begin{array}{l}
a_{N} \\
b_{N}
\end{array}\right)=P P^{\prime} y
$$

whence $x=P_{1} \ldots P_{N-1} P P^{\prime} y$.

(4) $b$ is not a nonnegative real number. As in (3), let $N$ be such that $r(\sec (\theta / N))^{N}$

$<1$, where $r=\left(|a|^{2}+|b|^{2}\right)^{1 / 2}$ and $\theta=\tan ^{-1}\left|\frac{b}{\mid a}\right|$. Let $\eta=\theta / N$ and $\theta_{j}=\theta-(j-1) \eta$ for $j=1,2, \ldots, N+1$. Let $a_{0}=a, b_{0}=b$, and, for $j=1,2, \ldots, N+1$, let

$$
a_{j}=r(\sec \eta)^{j-1} \cos \theta_{j} \frac{a_{j-1}}{\left|a_{j-1}\right|}
$$

and

$$
b_{j}=r(\sec \eta)^{j-1} \sin \theta_{j} \frac{b_{j-1}}{\left|b_{j-1}\right|} .
$$

As before, let $P_{j}=P\left(\theta_{j}, \arg a_{j-1}-\arg b_{j-1}\right)$ be the projection onto the subspace generated by $\left(\cos \theta_{j} \frac{a_{j-1}}{\left|a_{j-1}\right|} \frac{\left|b_{j-1}\right|}{b_{j-1}} \sin \theta_{j}\right)^{t}, j=1,2, \ldots, N$, or, equivalently, by $\left(\begin{array}{l}a_{j} \\ b_{j}\end{array}\right)$. It is easily seen that $a_{1}=a, b_{1}=b, a_{N+1}=r(\sec \eta)^{N} \frac{a_{N}}{\left|a_{N}\right|}$, $b_{N+1}=0$ and $\left(\begin{array}{l}a_{j} \\ b_{j}\end{array}\right)=P_{j}\left(\begin{array}{l}a_{j+1} \\ b_{j+1}\end{array}\right)$ for $j=1,2, \ldots, N$. Hence we have $x=$ $P_{1} P_{2} \ldots P_{N}\left(\begin{array}{l}a_{N+1} \\ b_{N+1}\end{array}\right)$. Since $\left|a_{N+1}\right|^{2}+\left|b_{N+1}\right|^{2}=r^{2}(\sec \eta)^{2 N}>0,\left(\begin{array}{l}a_{N+1} \\ b_{N+1}\end{array}\right)=Q_{1} \cdots Q_{m} y$ for some projections $Q_{1}, \ldots, Q_{m}$ by (3). We conclude that $x=P_{1} \cdots P_{N} Q_{1}$ $\cdots Q_{m} y$ as asserted.

The next lemma says that in the factorization of $2 \times 2$ matrices, the number of projection factors may be arbitrarily large.

Lemma 3.6. For any $m \geq 2$, let

$$
S_{m}=\left(\begin{array}{cc}
0 & \left(\cos \frac{\pi}{2 m}\right)^{m} \\
0 & 0
\end{array}\right)
$$

Then $S_{m}$ is the product of at least $m+1$ projections.

Proof. For $j=1,2, \ldots, m+1$, let $\theta_{j}=(j-1) \pi / 2 m$, and $P_{j}=P\left(\theta_{j}, 0\right)$. Since $P_{1}=\left(\begin{array}{ll}1 & 0 \\ 0 & 0\end{array}\right)$ and $P_{m+1}=\left(\begin{array}{ll}0 & 0 \\ 0 & 1\end{array}\right)$, a little computation yields that

$$
P_{1} P_{2} \ldots P_{m+1}=\left(\begin{array}{cc}
0 & \prod_{j=1}^{m} \cos \left(\theta_{j+1}-\theta_{j}\right) \\
0 & 0
\end{array}\right)=\left(\begin{array}{cc}
0 & \left(\cos \frac{\pi}{2 m}\right)^{m} \\
0 & 0
\end{array}\right)=S_{m} \text {. }
$$

To prove the minimality of $m+1$, assume that $S_{m}=Q_{1} Q_{2} \cdots Q_{k+1}$, where $k<m$ and the $Q_{j}$ 's are projections $\neq 1$. By Lemma 3.4, we may take 
$Q_{1}=\left(\begin{array}{ll}1 & 0 \\ 0 & 0\end{array}\right)$ and $Q_{k+1}=\left(\begin{array}{ll}0 & 0 \\ 0 & 1\end{array}\right)$. Since each $Q_{j}, j=2, \ldots, k$, is of the form $P\left(\theta_{j}, \alpha_{j}\right)$ for some $-\frac{1}{2} \pi \leq \theta_{j}<\frac{1}{2} \pi$ and $\alpha_{j}$, carrying out the multiplications in $S_{m}=Q_{1} Q_{2} \cdots Q_{k+1}$ and taking the absolute values of the resulting quantities we obtain $(\cos (\pi / 2 m))^{m} \leq \prod_{j=1}^{k}\left|\cos \eta_{j}\right|$, where $\eta_{j}=\theta_{j+1}-\theta_{j}$ if $\theta_{j}$ and $\theta_{j+1}$ are in the same quadrant and $\theta_{j+1}+\theta_{j}$ otherwise, and $\theta_{1}=0, \theta_{k+1}=\frac{1}{2} \pi$. Since $\cos x$ is an even function of $x$, we may suitably add a " + " or " - " sign in front of each $\eta_{j}$ such that their algebraic sum equals $\frac{\pi}{2}$. Thus Lemma 3.3(2) is applicable and we infer that the right hand side of the above inequality is no greater than $(\cos (\pi / 2 k))^{k}$. It follows that $(\cos (\pi / 2 m))^{m} \leq(\cos (\pi / 2 k))^{k}$. This contradicts Lemma 3.3(1) since $k<m$. The proof is complete.

Proof of Sufficiency in Theorem 3.1. Assume that $S$ is a singular strict contraction. Let $S=A B$ be its polar decomposition, where $A$ is a partial isometry and $B=\left(S^{*} S\right)^{1 / 2}$ is positive semidefinite with $\operatorname{rank} A=\operatorname{rank} B=\operatorname{rank} S$ (cf. [4, Problem 134]), and let $\alpha$ be a positive number satisfying $\|S\|<\alpha<1$. Since $S=(\alpha A)\left(\frac{1}{\alpha} B\right)$ and both $\alpha A$ and $\frac{1}{\alpha} B$ are singular strict contractions, to complete the proof we need only decompose these two factors into projections.

We first consider $\alpha A$. Let $A=\left(\begin{array}{c}A_{1} \\ A_{2}\end{array}\right)$ with respect to the decomposition ran $A^{*} \oplus \operatorname{ker} A$. We may assume that $A_{1}$ is lower triangular. Next express $\alpha A$ in column vectors as $\alpha A=\left(a_{1} \cdots a_{k} 0 \cdots 0\right)$, where $k=\operatorname{rank} A^{*}$. Since $A$ is a partial isometry, $a_{j}$ 's are mutually orthogonal with norms less than 1 . For $j=1,2, \ldots, k$, let

$$
e_{j}=\left(0 \cdots 0{ }_{j \mathrm{th}} \quad \cdots 0\right)^{t} .
$$

Since $e_{1}$ and $a_{1}$ are both orthogonal to $a_{2}, \ldots, a_{k}$ and $\left\|a_{1}\right\|<1=\left\|e_{1}\right\|$, by Lemma 3.5 we may transform $e_{1}$ to $a_{1}$ by a sequence of projections $P_{1}, \ldots, P_{n_{1}}$ while preserving $a_{2}, \ldots, a_{k}$, that is, $\alpha A=P_{1} \cdots P_{n_{1}}\left(e_{1} a_{2} \cdots a_{k} 0 \cdots 0\right)$. Repeating the argument, since $e_{2}$ and $a_{2}$ are orthogonal to $e_{1}, a_{3}, \ldots, a_{k}$, there are projections $P_{n_{1}+1}, \ldots, P_{n_{2}}$ such that $\left(e_{1} a_{2} \cdots a_{k} 0 \cdots 0\right)=P_{n_{1}+1} \ldots P_{n_{2}}$ $\left(e_{1} e_{2} a_{3} \cdots a_{k} 0 \cdots 0\right)$. In $k$ steps, we obtain that $\alpha A=P_{1} \cdots P_{n_{k}}\left(e_{1} \cdots e_{k} 0 \cdots 0\right)$ as a product of $n_{k}+1$ projections.

The factorization of $(1 / \alpha) B$ is even easier. Assuming that $\frac{1}{\alpha} B$ is diagonal, we may proceed as before since the column vectors of $\frac{1}{\alpha} B$ are mutually orthogonal. This proves the factorization of $S$.

To prove the assertion for the number of factors, let $T_{m}=I_{n-2} \oplus S_{m}$, where $I_{n-2}$ denotes the identity matrix of size $n-2$ and $S_{m}(m \geq 2)$ is the $2 \times$ 2 matrix as in Lemma 3.6. If $T_{m}=P_{1} P_{2} \cdots P_{k+1}$ is the product of $k+1$ projections, then, by Lemma 3.4, we may assume that $P_{k+1}=I_{n-2} \oplus\left(\begin{array}{ll}0 & 0 \\ 0 & 1\end{array}\right)$. Let $e_{i}=\left(\begin{array}{lllll}0 & \cdots & 1 & 0 & \cdots\end{array}\right)^{t}$ be the $i$ th column of $T_{m}$ and also of $P_{k+1}, i=$ $1,2, \ldots, n-2$. From $T_{m}=P_{1} P_{2} \ldots P_{k+1}$, we have $e_{i}=P_{1} P_{2} \cdots P_{k} e_{i}$. An argument as in the proof of the necessity part yields that $P_{j} e_{i}=e_{i}$ for all $i$ and $j$. Hence $P_{j}=I_{n-2} \oplus P_{j}^{\prime}$ for some $2 \times 2$ projection $P_{j}^{\prime}$, and we have 
$S_{m}=P_{1}^{\prime} P_{2}^{\prime} \cdots P_{k+1}^{\prime}$. It follows from Lemma 3.6 that $k \geq m$ completing the proof.

\section{REFERENCES}

1. C. S. Ballantine, Products of idempotent matrices, Linear Algebra Appl. 19 (1978), 81-86.

2. L. Brown, Almost every proper isometry is a shift, Indiana Univ. Math. J. 23 (1973), 429-431.

3. I. Erdelyi, Partial isometries closed under multiplication on Hilbert spaces, J. Math. Anal. Appl. 22 (1968), 546-551.

4. P. R. Halmos, A Hilbert space problem book, 2nd ed., Springer-Verlag, New York, 1982.

5. H. Radjavi and J. P. Williams, Products of self-adjoint operators, Michigan Math. J. 16 (1969), 177-185.

6. B. Sz.-Nagy and C. Foiaş, Harmonic analysis of operators on Hilbert space, North-Holland, Amsterdam, 1970.

Department of Mathematics, National Cheng Kung University, Tainan, Taiwan, RePUBLIC OF CHINA

Department of Applied Mathematics, National Chiao Tung University, Hsinchu, TaiWAN, REPUBLIC OF CHINA 